\title{
Introduction to the Special Issue on the Evolution and Development of the Universe
}

\author{
Clément Vidal
}

(C) Springer Science+Business Media B.V. 2010

In 2007, John M. Smart and I entered in contact discussing issues about universal change and broad cosmological and futuristic views. We noticed that scholars studying the cosmos where mainly into theoretical physics. It is of course an indispensable approach, but it does not strongly connects with life, intelligence and technology. Yet, we were also aware of dispersed insights in cosmology, theoretical and evolutionary developmental (evo-devo) biology and complexity sciences, which are providing ways to understand our universe within a broader framework.

We thought that these results and hypotheses deserved to be explored, criticized, and analyzed by an international interdisciplinary research community which we set up in 2008: 'Evo Devo Universe (EDU)'. Such a framework promises to advance our understanding of both unpredictable "evolutionary" processes and predictable "developmental" processes at all scales, including the human scale. I welcome any researcher interested in these topics to join the research community!

I wish to clarify that the expression "evo devo universe" does not imply that we chose to naively see the universe as a living system. It is more an invitation to consider the general mechanisms of biological processes (especially evolution and development) at universal scales. As I argue in my paper (to be published in Part III), analogies are never proofs, they mainly provide highly valuable heuristics to formulate new hypotheses and theories.

The Conference on the Evolution and Development of the Universe. John and I first started to work actively on building the EDU website in 2008. ${ }^{1}$ However, we understood that having a website and a virtual community was not enough. To effectively collaborate, human beings still need to meet in flesh. We thus focused our energy into setting up "The First International Conference on the Evolution and Development of the Universe" project, which was held at the École Normale Supérieure, Paris, 8-9 October 2008. ${ }^{2}$

\footnotetext{
1 http://www.evodevouniverse.com.

2 EDU 2008, http://evodevouniverse.com/wiki/Conference_2008.
}

C. Vidal $(\bowtie)$

Free University of Brussels (VUB), Brussels, Belgium

e-mail: clement.vidal@philosophons.com 
This special issue is a selection of papers presented at the conference, plus three other papers by Crane, Heylighen and Salthe. This volume is also the result of time and efforts from many referees (about 40 referee reports in total). I admire and endorse Richard Gordon's courage and position to refuse anonymous refereeing. I find the arguments for non-anonymous refereeing very compelling. Thomas Durt and Nicolás Lori also engaged in a transparent peer-review, which was long and sometimes difficult. I think that referees should be more recognized in this indispensable task for scientific activity. It is not normal that they do it on a voluntary basis and almost without recognition. A system to academically and publicly recognize this effort is yet to be invented.

General introduction to the papers. What follows is a general overview of the different papers constituting this special issue, which is divided into three parts. This first volume presents Part I with papers in physics and cosmology (Nottale; Greben; Durt; Lori \& Blin). Part II will present biology papers (Jagers op Akkerhuis; Ekstig; Chaline) and Part III philosophy papers (Heylighen; Salthe; Crane; Vidal; Stewart). The three parts will be published in different issues of Foundations of Science.

Scale Relativity. Laurent Nottale presents a review of Scale Relativity (ScR), a natural extension of Einstein's general relativity. The principle of relativity has been successfully applied to position, orientation and motion and is at the core of all physical theories. However, problems related to scales are ubiquitous in science. In particular, physics struggles for decades to connect in a meaningful way quantum theory (microphysics) with classical physics (macrophysics). In a similar manner, relating our knowledge of macrophysics to the cosmological scale leads to arduous problems in cosmology, for example about dark matter or vacuum energy density.

Scale Relativity proposes "to extend theories of relativity by including the scale in the very definition of the coordinate system, then to account for these scale transformations in a relativistic way." How is it possible? And why did Einstein not found this extension before? As often in the history of physics, part of the answer lies in the mathematical tools.

Einstein struggled years to develop the general relativity theory of gravitation, because it involved non-Euclidean geometries. These geometries are (or were) counter-intuitive to manipulate and understand, and they were not used in physics before. Similarly, ScR uses a fundamental mathematical tool to deal with scales: fractals. Including explicitly scale transformations in the equations leads to an extension of general relativity (i.e. it includes its previous results) with the construction of a fractal space-time theory. As with non-Euclidean geometries were new for Einstein, fractals were only studied in depth by Mandelbrot in the 1950s (although they were known by mathematicians much before; e.g. with Georg Cantor's triadic set).

This simple yet fundamental approach generates a proliferation of results, which are both theoretical and with concrete applications and validated predictions. Let us mention of few of them. A new light on quantum mechanics can be thrown, since it is possible to derive postulates of quantum mechanics with ScR. ${ }^{3}$ Furthermore, a macroscopic Schrödinger equation is derived, which brings statistical predictability characteristic of quantum mechanics into other scales in nature. For example, the position of exoplanets can be predicted in a statistical manner. The theory predicts that they have more chances to be found at such or such distance from their star. On cosmological scales, ScR also predicted with great precision the value of the cosmological constant (see section 3.1.2).

\footnotetext{
${ }^{3}$ Nottale, L., \& Celerier, M. N. (2007). Derivation of the postulates of quantum mechanics from the first principles of scale relativity. Journal of Physics A-Mathematical and Theoretical, 40(48), 14471-14498. http:// arxiv.org/abs/0711.2418.
} 
Models constructed with the general idea of relativity of scales bring new insights not only in physics, but also in earth sciences, history, geography and biology. All these are reviewed in Nottale's paper.

Scale Relativity is a fundamental approach which has consequences for nearly all sciences. It suggests that cosmology, fundamental particle physics, structure formation, biology, geology, economy and other fields might be approached with tools derived from the same few principles exposed in this paper. Although, as Nottale explains, a lot of work still has to be done, the exposed vision is extraordinarily far reaching and inspiring. For these reasons, I am delighted to deliver Laurent Nottale the EDU 2008 Best Paper Award.

Jean Chaline's paper (to be published in Part II) applies ScR principles to biological and paleontological data. He shows in his paper that log-periodic behaviors of acceleration or deceleration can be applied to branching macroevolution and to the time sequences of major evolutionary leaps. This includes the global tree of life, sauropod and theropod dinosaurs postural structures, North American fossil equids, rodents, primates, echinoderms clades and human ontogeny.

Greben's Cosmological Model. Jan Greben presents a cosmological model where the vacuum energy dominates the universe. He claims that this model avoids the horizon and cosmological constant problems, and also provides a possible explanation for dark matter. Greben also includes a model of the evolution of the early universe, which describes the formation of elementary particles from a supposed classical state with perfect symmetry and zero entropy.

Quantum Darwinism. Thomas Durt's paper tackles a very basic question about our nature as observers. Why is our representation of the world classical although at smallest scales, it is quantum? Durt reminds us that "millions of years of evolution modelled our vision of the world, leading us to become blind to aspects of it that are not advantageous from the point of view of the acquisition of useful information." He proposes along the lines of Zurek's Quantum Darwinism approach that the basis in which we measure the external world obeys an optimality principle that results from a biological selection mechanism: "the fittest is the best-informed." More precisely, he aims at establishing the identity between "classical islands" and our cognitive representation of what elementary particles are.

Nicolás Lori and Alex Blin's paper constitutes another application of Quantum Darwinism, yet in cosmology. To formalize Quantum Darwinism and cope with the random extinction of information, they define and distinguish between Formal Axiomatic Systems (FAS) and Darwinian Axiomatic Systems (DAS). After reminding us that "Gödel's incompleteness theorems showed that a non-trivial axiomatic system cannot be both complete and consistent" they add that "the FAS is the choice for consistency and the DAS is the choice for completeness". This approach is then applied to cosmic inflation. This constitute a very original reflection yet involving speculative hypotheses like cosmic inflation or baby universes.

Life and Complexity. In his paper, Gerard Jagers op Akkerhuis conducts a reflection on the definition of life. He first constructs a broader context, a "theory of life", from which he derives a definition of life. He uses hierarchy theory to this end, and defines a ranking called the "operator hierarchy". From this hierarchical perspective, he argues that to define life, construction is more important than metabolism, growth or reproduction.

Börje Ekstig argues that there is an acceleration of complexity in evolution. His paper presents a model integrating evolution on large time scales, and the development of an individual. He suggests some methods to estimate the growth of complexity in evolution, and also connects biological and cultural evolution. 
Philosophy and Big Questions. Francis Heylighen analyzes our most fundamental scientific concepts: time and causality. He explains how the concept of self-organization can be applied in this context. Starting from a random graph of events, he shows how a transitive closure can transform it into a partial order relation of precedence and thus generate a causal structure.

Stanley Salthe's reflection presents a broad and challenging outlook in natural philosophy. He argues that Aristotelian causal analysis, and especially final causes, can be helpful for dealing with complex systems. More precisely, he takes a developmental perspective to model our world, and uses a "specification hierarchy" to describe the emergence of systems of higher levels in the universe.

Louis Crane's paper explores philosophical implications of a supposed quantum theory of gravity. He builds on Lee Smolin's Cosmological Natural Selection (CNS) and conjectures that future civilizations will want to create black holes.

In my paper, I explore computational and biological analogies to address the fine-tuning issue in cosmology. I show that current solutions are not actually satisfying, to motivate other approaches. First, I analyze what are physical constants from a physical perspective. Then, I explore computational and biological analogies to tackle this issue, and propose an extension of CNS, stimulated by ideas from Crane and other authors. Inspired by a biological analogy, I name this extension of CNS "Cosmological Artificial Selection".

John Stewart takes a broad evolutionary view on the cosmos in his paper. After summarizing the arguments supporting the proposition that biological evolution has a trajectory, he attempts to extend it to the universe as a whole. He also builds on the work of Crane and other authors to explore the idea that "our universe and the evolution of life within it is a developmental process that has itself been shaped by evolutionary processes of even wider scale". He further argues that at a particular point, evolution will continue to advance only if we decide to advance the evolutionary process intentionally.

I hope the reader will be inspired by the insights gathered in this special issue to further build an even more comprehensive view on the cosmos.

\section{Acknowledgments}

John Smart and I would like to thank the following people. First, we would like to dedicate this special issue to the memory of Peter Winiwarter, who tragically ended his life this summer 2009. We have always been very grateful for his active help and enthusiasm, especially for the first steps of EDU community building. He will always remain in our memories. I warmly thank Alain Prochiantz for his trust and support for organizing this conference; JeanPierre Luminet for his early interest and The Complex Systems Institute, Paris (ISC-PIF) for setting up this interdisciplinary "call for ideas" and for funding EDU2008. We also very much appreciated Georges Ellis' early endorsement of the EDU project, along with 45 other researchers from all disciplines. ${ }^{4}$ A special thank is due to the EDU scientific board, ${ }^{5}$ James A. Coffman, James N. Gardner, Carlos Gershenson, Richard Gordon, Stevan Harnad, Francis Heylighen, David Holcman, Nicolás Lori, Laurent Nottale for their numerous advices. We also thank Mehdi Khamassi, Arnaud Blanchard, Mariam Chammat, Elizabeth Molière, Lina Boitier and Dominique Borg for their enthusiastic help during the conference. I am very grateful for the remarkable work anonymous referees did with their critical assessment and

\footnotetext{
4 http://evodevouniverse.com/wiki/People.

5 http://evodevouniverse.com/wiki/Scientific_Board.
} 
in their help to select and improve the papers. I am immensely grateful to my colleague and friend John Stewart for his efforts of coming from Australia to attend the conference and for sharing such inspiring insights and visions on evolution. I am most grateful to my colleague and friend John Smart for his energy, perseverance, insights, vision, and for being an amazing colleague to work with. It has been an empowering adventure to work with him, and I would like to express my deepest gratitude for everything I learn thanks to him. 